\title{
Information modeling and reengineering for product development process
}

\author{
Renbin Xiao ${ }^{1 *}$, Tinggui Chen ${ }^{2}$, Zhenwu Tao ${ }^{2}$ \\ ${ }^{1}$ School of Mangement, Huazhong University of Science and Technology, Wuhan 430074, Hubei, P. R. China \\ ${ }^{2}$ CAD Center, Huazhong University of Science and Technology, Wuhan 430074, Hubei, P. R. China
}

(Received November 3 2006, Accepted January 11 2007)

\begin{abstract}
Design process is an important stage in product development. Information model is a useful tool in analyzing and improving complex design process. It can facilitate to rebuild the process through visualizing information flow. Owing to the disadvantages of describing complex design process with data flow diagram (DFD), the extended DFD with powerful description ability is put forward. After the extended DFD is transformed into information model, the present optimal design flow is evaluated and mapped to design structure matrix (DSM). Based on DSM, the reengineering of design process can help manage tasks in concurrent design process. Interpretative structural modeling (ISM) method is adopted to realize the partitioning programming of DSM. Grey theory and work transformation matrix (WTM) are used to find new sequence of the coupled block. Eventually, one case study is employed to illustrate the method and the result validates that it can shorten the time and guarantees the quality and function of design process.
\end{abstract}

Keywords: extended data flow diagram, information modeling, design structure matrix, grey theory, work transformation matrix

\section{Introduction}

Product development is a complex process. It includes design and manufacture stages. About $80 \%$ of the total cost in product development is consumed in design stage. So, the paper discusses design process with emphasis on flow diagram. In general, information flow of design process is regard as a formal model to describe design tasks. That formal model is diverse in different systems. As good modeling tools, directed graph $^{[3]}$ and Petri-Nets ${ }^{[4]}$ are widely used. When the original model was built, it only considered the whole information of the design process rather than the optimization relationship between the sub-tasks in an abstract model. So, model reengineering should be carried on after information model of the design process is set up. The present methods include process reengineering based on IDEF (ICMA Definition) series (such as IDEF0, IDEF1, IDEF2, IDEF3) $)^{[5]}$, process optimization based on conditional directed $\operatorname{graph}^{[12]}$, process analyzing and reengineering by combining flow analysis methods with $\mathrm{IDEF}^{[13]}$. However, when several optimization methods mentioned above were used, we should arrange task executive order and know of relevancy intensity among tasks before analyzing and reengineering the design process, and original description of the design process should be accurate and complete. All of those are too difficult to be realized.

Yu Wang ${ }^{[8]}$ proposed a method based on Data Flow Diagram (DFD) and design structure matrix $(\mathrm{DSM})^{[7]}$. But there are some disadvantages in DFD. For instance: (1) Simple relationship. Most of DFD can only describe the sequential relationship. However, there also exist concurrent relationship, selection relationship, exclusive relationship, etc. in design process. DFD cannot describe those relationships completely. (2) Scattered structure and difficulty with operating on computer. Since DFD model is described in a graphical and illogical way, it is inconvenient to be operated on computer. Furthermore, if information flows are complex

\footnotetext{
* Corresponding author. Tel.: +86-27-87547405; E-mail address: rbxiao@ mail.hust.edu.cn.
} 
or information content is great, DFD model will be out of order. Therefore, an extend DFD is put forward to describe complex design process. And we are able to obtain a sequential presentation of design process after analyzing the flows hidden in the extended DFD and mapping it to DSM. Since an extended DFD includes many relations, this paper takes Fuzzy Evaluation method ${ }^{[11]}$ to evaluate the possible flows.

In addition, in partitioning programming of DSM, initial matrix is transformed into an almost triangular form firstly, sets of coupled tasks are found out secondly, and uncoupled tasks are arranged eventually. In process of disposing coupling of DSM, a method combining tearing with iteration is proposed. The key step in this approach is to find out the weakest point in information-dependent loop to cut off and then the sub-block is obtained. Using work transformation matrix $(\mathrm{WTM})^{[6]}$, we can calculate the total work of it.

This paper is organized as follows. In section 2, the whole research framework is proposed. In section 3 , different information flows of product development process are built and present optimal flow by evaluating the possible flows is acquired. In section 4, reengineering of product development process based on DSM including partition programming and disposing coupling programming is discussed. In section 5, an example is employed to justify the proposed method. Section 6 is some conclusions reached by this paper.

\section{Research framwork}

According to the above analysis, the research steps are brought forward. First, the information modeling is carried on through describing the requirement information with DFD and transforming the DFD into design information flow. Second, a current optimal flow is obtained by evaluating the potential design flows. Finally, after mapping the optimal information flow into DSM, the reengineering of the design process is achieved through partition and disposing coupling of DSM. Fig. 1 shows the research framework.

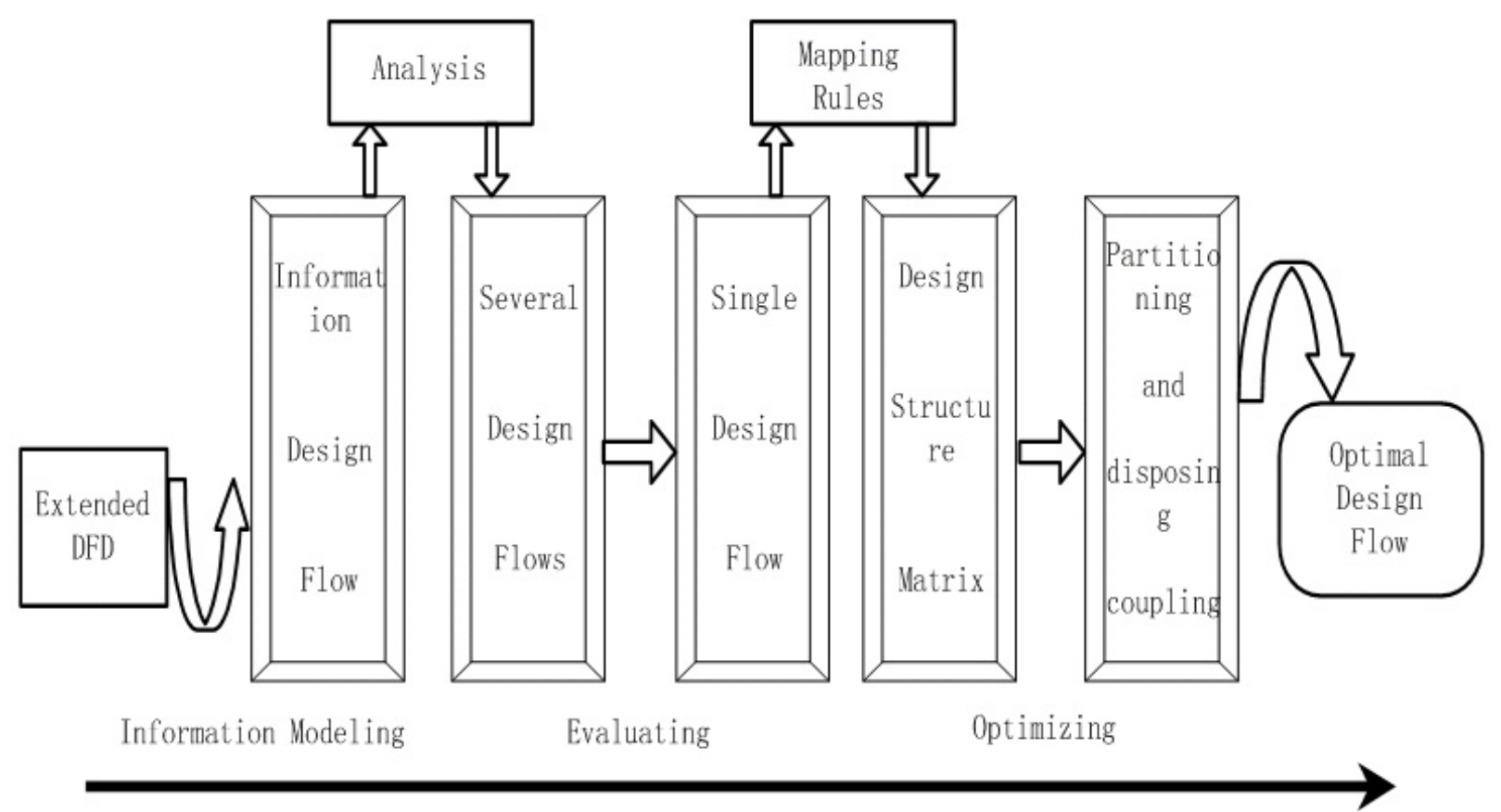

Fig. 1. Research framework

\section{Information modeling based on extended DFD}

Extended DFD is able to include several relationships. In this paper, three basic relationships: concurrent relationship, "or" relationship and conditional selection relationship are considered. In order to facilitate analysis, we take the design process in certain factory for an example. Fig. 2 is corresponding extended DFD. 
When there are two or more inputs in a process of extended DFD, a given symbol is used to explain the relationships among those inputs. The symbol "AND" represents the concurrent relationship of the inputs. "OR" represents the "or" relationship. And "CON" represents the conditional selection relationship.

The extended DFD can easily describe the product development process in a graphic way. But it is inconvenient to be realized on computer. So it is necessary to transform the extended DFD into design information flow and then map it to DSM. DSM is an algebraic method that can be conveniently realized on computer. Fig.3 is the design information flow corresponding to Fig.2. In Fig.3, there are ten tasks corresponding to seven processes, two sources and one host in Fig.2, respectively. 1-custom requirement, 2-market sale, 3plan specification, 4-project confirmation, 5-sale planning, 6-new product development, 7-performance testing, 8-product improvement, 9-old product improvement, 10-product sale. And the mark " $\&$ " in the 3 rd node represents concurrent relationship. Mark "o" in the 4th node represents "or" relationship. Mark "E" in the 7th node represents conditional selection relationship.

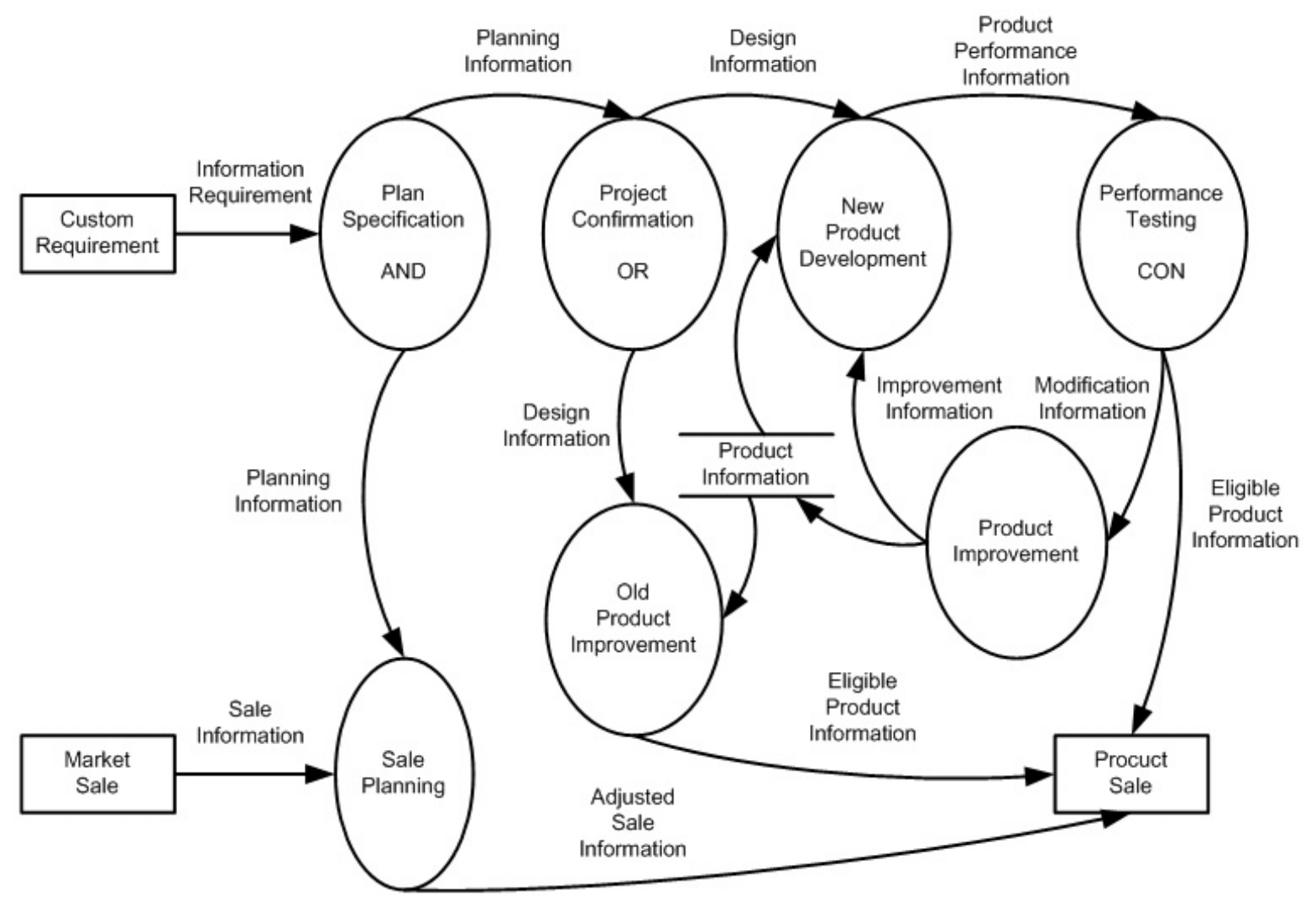

Fig. 2. Extended DFD of design flow in a factory

In Fig. 3, design information starts from task 1 and task 2 at the same time. After task 3 is accomplished, task 4 and 5 are executed simultaneously and then the information of task 5 goes directly to task 10 . There are three possible paths after task 4 completed. The first path is from task 4 to task 6 . The second path is from task 4 to task 9 . The third path is from task 4 to task 6 and 9 simultaneously. Whichever path is selected, task 10 is followed after task 9 is executed. Notice that task 7 is a conditional selection relationship. If the information coming from task 6 satisfies the condition of task 7, task 10 is executed, or task 8 will be carried on and task 6 will be repeated. That loop will not stop until the information from task 6 satisfies the condition. $W$ is denoted as the design flow. There are five possible paths listed as follows.

$$
\begin{aligned}
& W_{1}=\{(2,5),(1,3),(3,5),(5,10),(3,4),(4,9),(9,10)\} \\
& W_{2}=\{(2,5),(1,3),(3,5),(5,10),(3,4),(4,6),(6,7),(7,10)\} ; \\
& W_{3}=\{(2,5),(1,3),(3,5),(5,10),(3,4),(4,6),(6,7),(7,8),(8,6),(6,7),(7,10)\} ; \\
& W_{4}=\{(2,5),(1,3),(3,5),(5,10),(3,4),(4,9),(4,6),(6,7),(9,10),(7,10)\} ; \\
& W_{5}=\{(2,5),(1,3),(3,5),(5,10),(3,4),(4,9),(4,6),(6,7),(7,8),(8,6),(6,7),(9,10),(7,10)\} .
\end{aligned}
$$




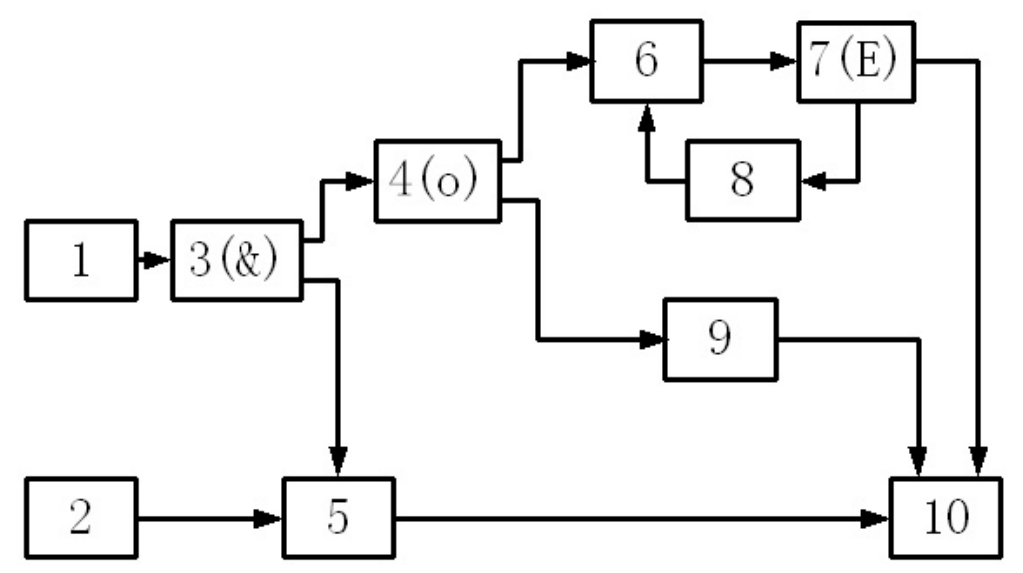

Fig. 3. Design information flow

The original extended DFD is corresponding to five design flows, which can be mapped to five DSMs. The evaluation of design information flows relates to many experiences and much knowledge, such as how to plan the index domain, how to evaluate the design schemas evaluation, how to determine the weight of every indexand so on. Considering the characteristics of the design process, the integrated evaluation of design information flows can be made from design time, product performance, utilizing rate of device, product repertory, produce efficiency, and investment. Finally, a current optimal flow $W_{5}$ is acquired using fuzzy evaluation. Since an extended DFD can be transformed to a directed graph and the transpose of the adjacency matrix of the directed graph is DSM, so each element in DSM denotes one task in the extended DFD. Fig. 4 shows the top-level DSM corresponding to $W_{5}$.

\begin{tabular}{|l|c|c|c|c|c|c|c|c|c|c|c|}
\hline & & 1 & 2 & 3 & 4 & 5 & 6 & 7 & 8 & 9 & 10 \\
\hline Custom Requirement & 1 & & & & & & & & & & \\
\hline Market Sale & 2 & & & & & & & & & & \\
\hline Plan Specification & 3 & 1 & & & & & & & & & \\
\hline Project Confirmation & 4 & & & 1 & & & & & & & \\
\hline Sale Planning & 5 & & 1 & 1 & & & & & & & \\
\hline New Product Development & 6 & & & & 1 & & & & 1 & & \\
\hline Performance Testing & 7 & & & & & & 1 & & & & \\
\hline Product Improvement & 8 & & & & & & & 1 & & & \\
\hline Old Product Improvement & 9 & & & & 1 & & & & & & \\
\hline Product Sale & 10 & & & & & 1 & & 1 & & 1 & \\
\hline
\end{tabular}

Fig. 4. The top-level DSM Corresponding to $W_{5}$ 


\section{Process reengineering based on DSM}

In Fig. 4, some elements in top-level DSM perhaps include several small sub-elements. Thus, a top-down method is usually taken to refine these elements in top-level DSM. Fig. 5 shows the final DSM corresponding to design information flow $W_{5}$.

\begin{tabular}{|l|l|l|l|l|l|l|l|l|l|l|l|l|l|l|l|l|l|l|l|}
\hline & & 1 & 2 & 3 & 4 & 5 & 6 & 7 & 8 & 9 & 10 & 11 & 12 & 13 & 14 & 15 & 16 & 17 & 18 \\
\hline Requirement Analysis & 1 & & & & & & & & & & & & & & & & & & \\
\hline Sale Analysis & 2 & & & & & & & & & & & & & & & & & & \\
\hline Sale Project & 3 & 1 & & & & & & & & & & & & & & & & & \\
\hline Product Sale & 4 & & & & & 1 & & & 1 & & & & & 1 & & & & & \\
\hline Sale Planning & 5 & & 1 & 1 & & & & & & & & & & & & & & & \\
\hline Design Planning & 6 & 1 & & & & & & & & & & & & & & & & & \\
\hline New Product Design & 7 & & & & & & & & & & 1 & 1 & & & & 1 & 1 & 1 & \\
\hline New Product Produce & 8 & & & & & & & & & & & & & & 1 & & 1 & 1 & 1 \\
\hline Performance Testing & 9 & & & & & & & & 1 & & & & & & & & & 1 & \\
\hline Design and Produce Project & 10 & & & & & & 1 & & & & & & & & & & & \\
\hline New Product Improvement & 11 & & & & & & & 1 & & 1 & & & & & & 1 & 1 & \\
\hline Old Product Improvement & 12 & & & & & & & & & & 1 & & & 1 & & 1 & & \\
\hline Old Product Produce & 13 & & & & & & & & & & & & 1 & & 1 & & & \\
\hline Requirement of Time & 14 & & & & & & 1 & & & & & & & & & & & \\
\hline Requirement of Performance & 15 & 1 & & & & & & & & & & & & & & & & \\
\hline Technology Analysis & 16 & & & & & & 1 & & & & & & & & & 1 \\
\hline Performance Analysis & 17 & & & & & & 1 & & 1 & & & & & & & 1 \\
\hline Drawing of Produce Project & 18 & & & & & & 1 & & & & & & & & & \\
\hline
\end{tabular}

Fig. 5. Thinned DSM from the peak DSM

Notice that the sequence of tasks is random in Fig.5. Comparing top-level DSM, the relationships between tasks in refined DSM are not clear. The reason mostly lies in that modularized expression covers too much information. Once the modules are refined, the relationships between them become quite complex. There are three basic relationships ${ }^{[10]}$ : sequential mode (Fig. 6 a), parallel mode (Fig. 6 b) and coupled mode (Fig. 6 c). In parallel mode, elements are irrelevant, they can execute at the same time. In sequential mode, the influences between elements are directed. In coupled mode, the influences between elements are interacted, which consist of information loop. It is the information loop in coupled mode that leads to iterations of design activities and the delay of design period. So the purpose of reengineering is to reduce the time of iterations as possible as the process permits. Because the above DSM is based on activities, its reengineering can be realized by partitioning and disposing coupling of $\mathrm{DSM}^{[1]}$.

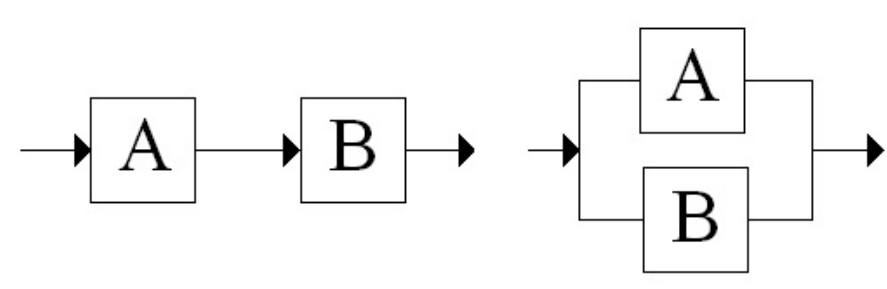

(a)

(b)

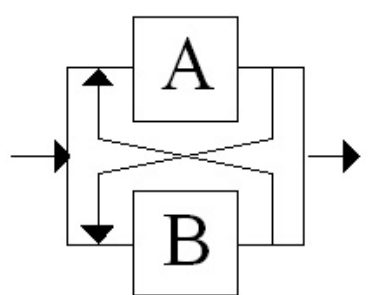

(c)

Fig. 6. Three connection forms among design task 


\subsection{Partition programming of DSM}

The upper-diagonal marks of DSM signify feedback and iteration of tasks. So the purpose of partition is to transform DSM into a lower triangular matrix. Interpretative Structural Modeling (ISM) method ${ }^{[9]}$ is adopted to realize the partitioning programming of DSM. There are two main steps: identifying the coupled tasks and arranging the ranks of the uncoupled tasks. We firstly introduce two definitions which will be used in partitioning programming.

Definition 1. Let $\boldsymbol{A}$ be the adjacency matrix of a DSM and let $I_{n}$ be the n-dimensional unit matrix, then the transitive closure of $\boldsymbol{A} \cup I_{n}$ is defined as the reachable matrix of this DSM, denoted as $\boldsymbol{R}$.

Definition 2. Let $\boldsymbol{R}$ be the reachable matrix of a DSM. After deleting the rows and columns whose all the components are equal to 0 in $\boldsymbol{R}$, the rest forms a new matrix $\boldsymbol{R}^{\prime} . \boldsymbol{R}^{\prime}$ is defined as the reductive matrix of DSM.

The steps of partitioning programming are as follows.

Step 1. Identify the coupled tasks.

Let $\mathbf{R}$ be the reachable matrix of a DSM, and let $\bigcap$ be the logic multiply between Boolean matrixes, denote $\mathbf{R} \bigcap \mathbf{R}^{T}=\left(\bar{r}_{i j}\right)_{n \times n}=\left(r_{1}, r_{2}, \cdots, r_{n}\right)^{T}$ where $r_{i}$ is a $n$-dimensional row vectorand let the set composed by any of the unequal $r_{i}$ be $\left\{r_{1}^{\prime}, r_{2}^{\prime}, \cdots, r_{m}^{\prime}\right\}(1 \leq m \leq n)$. Then:

(1) The number of coupled tasks in DSM is $m^{\prime}\left(m^{\prime} \leq m\right)$, where $m^{\prime}$ is the total number of row vector that has at least one component whose value is equal to 1 in $\left\{r_{1}^{\prime}, r_{2}^{\prime}, \cdots, r_{m}^{\prime}\right\}$.

(2) If $r_{i}^{\prime}$ is the row vector that has at least one component whose value is equal to 1 and all the components whose value is equal to 1 are $r_{i k_{1}}, r_{i k_{2}}, \cdots, r_{i k_{p}},(2 \leq p \leq n)$, then $\mathbf{C}=\left\{T_{i k_{1}}, T_{i k_{2}}, \cdots, T_{i k_{p}}\right\}$ is a coupled tasks set.

Step 2. Arrange the ranks of the uncoupled tasks.

Let $\mathbf{R}^{\prime}=\left(r_{i j}^{\prime}\right)_{q \times q}$ be the reductive matrix of a DSM. $\mathbf{R}^{\prime} \mathbf{E}_{l-1}=\left(r_{1}, r_{2}, \cdots, r_{m}\right)^{T}, l \geq 1, \mathbf{E}_{0}=$ $(1,1, \cdots, 1)^{T}, \mathbf{E}_{l}=\left(e_{1}, e_{2}, \cdots, e_{q}\right)^{T}$, where

$$
e_{i}= \begin{cases}0, & r_{i} \in\{0,1\} \\ 1, & r_{i} \notin\{0,1\}\end{cases}
$$

Then, for task $T_{i}, r_{i}=1$ is the necessary and sufficient condition of $T_{i}$ being the $l$ level task of DSM.

Theorem 2 can also be easily realized on computer to arrange the level of unsets of coupled tasks.

According to the above method, the partitioned DSM of design flow can be obtained in Fig. 7. In Fig. 7, the execution sequence of tasks becomes sequential. The rank of task indicates the priority level of all the tasks. Comparing with Fig. 5, the design process presents lower triangularization, and there are no large-scale or whole iteration.

\subsection{Strategies for disposing coupling}

A lower triangular form by partitioning is able to avoid a large-scale iteration, but loops in coupled blocks are still not disappearing. So it is necessary to break those loops and schedule them. For the sake of reducing the feedback and iteration caused by coupled information flow, we use a removing coupling method called tearing to make certain the original iteration sequence of coupled tasks through analyzing the relationships between tasks. The basic principle of tearing algorithm is to cut off the loops at the weakest point and to firstly execute the task with the least information-dependent intensity. Moreover, in order to quantify the informationdependent intensity, we introduce Grey theory ${ }^{[2]}$ to deal with the uncertainty of information. At the same time, Grey Relational Intensity (GRI) is used to quantify the interaction between tasks. For two tasks $T_{p}$ and $T_{q}$, the bigger the GRI between them is, the higher the information-dependent intensity is. The computing process of GRI is as follows.

Let $X_{i}=\left\{x_{i}(k)\right\}$ and $\left.X_{j}=\left\{x_{j}(k)\right\} k=1,2, \cdots, n ; i, j=1,2, \cdots, m\right)$ be the averaged fuzzy evaluation vector under the quality and function index of products, where $n$ is the number of index and $m$ is the number of coupled tasks. Then the Grey Relational Coefficient (GRC) between $X_{i}$ and $X_{j}$ under index $k$ is 


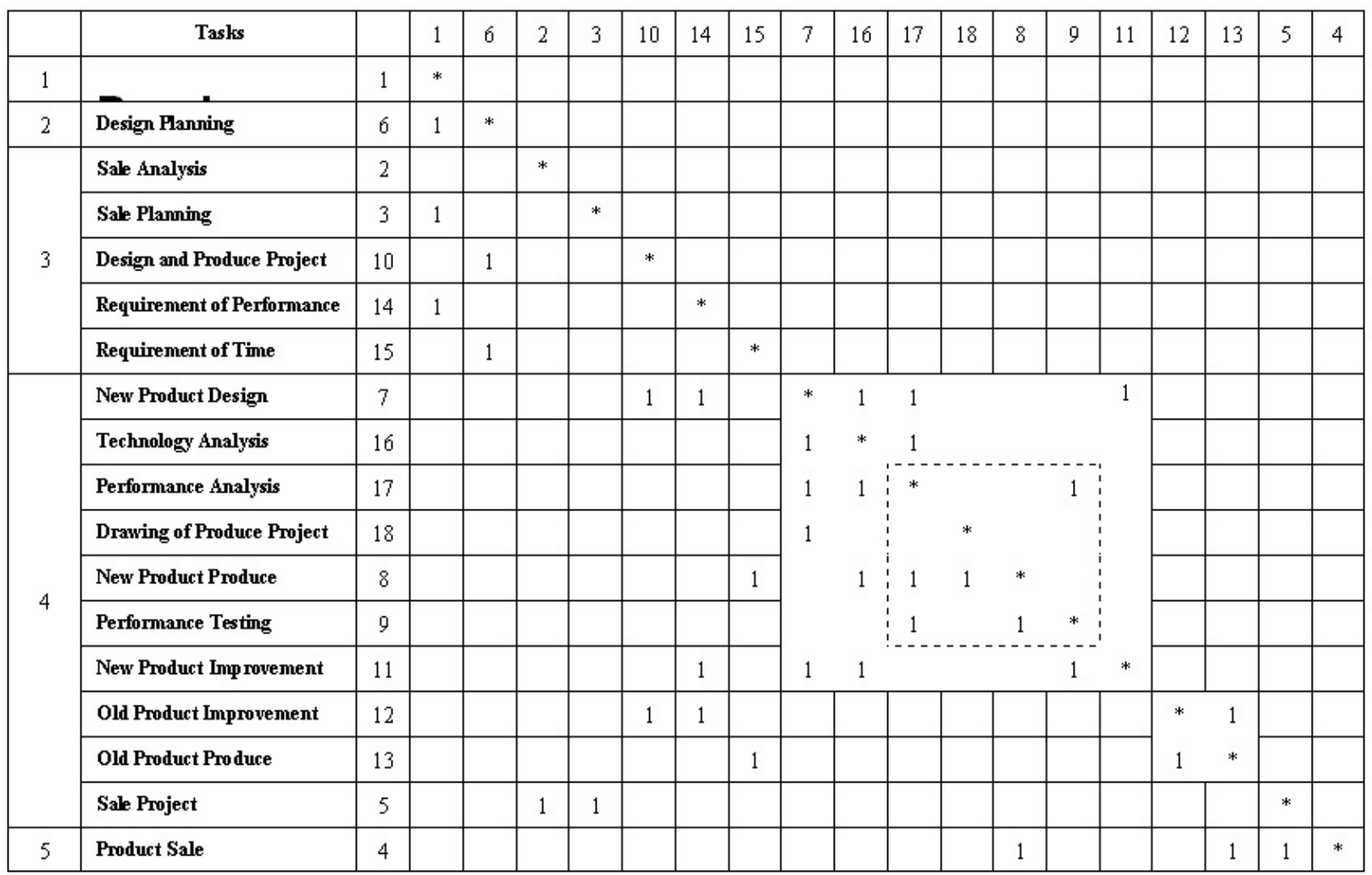

Fig. 7. Partitioned DSM

$$
\xi\left(x_{i}(k), x_{j}(k)\right)=\frac{\min _{i} \min _{j} \min _{k}\left|x_{i}(k)-x_{j}(k)\right|+\rho \cdot \max _{i} \max _{j} \max _{k}\left|x_{i}(k)-x_{j}(k)\right|}{\left|x_{j}(k)-x_{i}(k)\right|+\rho \cdot \max _{i} \max _{j} \max _{k}\left|x_{i}(k)-x_{j}(k)\right|},
$$

where $\rho$ is the recognition differential $\rho \in[0,1]$, generally $\rho=0.5 . X_{i}$ is defined as reference sequence and $X_{j}$ is defined as comparison sequence. Then the GRI between $X_{i}$ and $X_{j}$ under all the indexes is:

$$
r\left(X_{i}, X_{j}\right)=\frac{1}{n} \sum_{k=1}^{n} \xi\left(x_{i}(k), x_{j}(k)\right) .
$$

Since the pairwise comparison is used in the computation of GRI, the reference sequence is not unique and every sequence acts as both reference sequence and comparison sequence. This kind of grey relation is also defined as grey self-relation. The GRIs between tasks in sets of coupled tasks can be integrated into one Grey Self-relational Matrix:

$$
\mathbf{R}=\left[\begin{array}{cccc}
r\left(X_{1}, X_{1}\right) & r\left(X_{1}, X_{2}\right) & \cdots & r\left(X_{1}, X_{m}\right) \\
r\left(X_{2}, X_{1}\right) & r\left(X_{2}, X_{2}\right) & \cdots & r\left(X_{2}, X_{m}\right) \\
\vdots & \vdots & \vdots & \vdots \\
r\left(X_{m}, X_{1}\right) & r\left(X_{m}, X_{2}\right) & \cdots & r\left(X_{m}, X_{m}\right)
\end{array}\right]
$$

where $r\left(X_{i}, X_{i}\right)=1, r\left(X_{j}, X_{i}\right)=r\left(X_{i}, X_{j}\right), i, j=1,2, \cdots, m$. Based on $\mathbf{R}$, the task pair with minimum GRI can be found:

$$
\left(T_{p}, T_{q}\right)=\min _{i} \min _{j} r\left(X_{i}, X_{j}\right), p, q \in\{1,2, \cdots, m\}, i, j=1,2, \cdots, m .
$$

Formula (5) means that there is the lowest information-dependent intensity between task $T_{p}$ and $T_{q}$ under the product evaluation indexes.

In addition, because of producing quality loss of tearing algorithm, Smith and Eppinger ${ }^{[6]}$ apply an adding coupling approach and propose work transformation matrix (WTM) to predict slow and rapid convergence of 
iteration within a project, and predict the coupled features of the design problem which will require many iterations to reach a technical solution. However, the authors also point out the sufficient condition of the convergence of iteration in coupled block is that the entries either in every row or in every column of WTM sum to less than one. If a design process is not converging, it might be appropriate to abandon the project, or to adapt the specifications and restructure the problem. Consequently WTM has its own applicable condition.

Comparing these two approaches, we can find out that tearing is relatively easier and no condition constraint to apply, but often brings quality loss. On the other hand, using WTM to adding coupling is more accurate but must satisfy its convergence condition. Therefore, combining these two approaches is likely to be more scientific and effective. The essential notation is to ignore the weakest feedback marks by tearing in coupling in order to find the sub-blocks, and then use WTM to dispose them as long as they satisfy convergence condition. The more detailed process will be discussed in another paper. The idiographic steps of strategy for disposing coupling are as follows:

Step 1. Based on Fuzzy Evaluation method ${ }^{[4]}$, multi experts propose a domain of product evaluation index and compute the fuzzy evaluation vector of every task;

Step 2. Compute the Grey Self-relational Matrix of sets of coupled tasks by formula (1), (2) and (3) which will be described in the following text;

Step 3. Find out the task pair $\left(T_{p}, T_{q}\right)$ with the minimum GRI in Grey Self-relational Matrix. Cut off the information connection between them. Set the corresponding elements to 0 in DSM.

Step 4. Reprogram the coupled tasks according to Theorem 1 and 2. If there are still coupled tasks in DSM, return the rest sets of coupled tasks to Step 2; otherwise, go to Step 5.

Step 5. After tearing, ignore the weakest feedback marks. And according to the new sequence, find the sub-block in coupling and judge its convergence, and then apply WTM to calculate their workload. After that, take the sub-block as a simple element to make certain execution order. In doing so, the complex of the design process will be reduced.

\section{An example}

According to Fig. 7, suppose $T_{1}$ denotes the new product design, $T_{2}$ denotes the technology analysis, $T_{3}$ denotes the performance analysis, $T_{4}$ denotes the produce project drawing, $T_{5}$ denotes the produce of new product, $T_{6}$ denotes the performance test, and $T_{7}$ denotes the improvement of new product. The fuzzy evaluation vectors of $T_{1} \sim T_{7}$ are listed in Column 2 in Tab. 1 and their averaged sequences are listed in Column 3 in Tab. 1.

Table 1. The fuzzy evaluation vectors of coupled tasks and their averaged sequences

\begin{tabular}{ccc}
\hline Task & Fuzzy evaluation vectors & Averaged sequences \\
\hline$T_{1}$ & $(0.1,0.25,0.35,0.3)$ & $(0.4000,1.0000,1.4000,1.2000)$ \\
$T_{2}$ & $(0.2,0.2667,0.2667,0.2667)$ & $(0.8000,1.0668,1.0668,1.0668)$ \\
$T_{3}$ & $(0.187,0.333,0.25,0.23)$ & $(0.7480,1.3320,1.0000,0.9200)$ \\
$T_{4}$ & $(0.15,0.35,0.28,0.22)$ & $(0.6000,1.4000,1.1200,0.8800)$ \\
$T_{5}$ & $(0.22,0.22,0.28,0.28)$ & $(0.8800,0.8800,1.1200,1.1200)$ \\
$T_{6}$ & $(0.3,0.125,0.275,0.3)$ & $(1.2000,0.5000,1.1000,1.2000)$ \\
$T_{7}$ & $(0.28,0.42,0.15,0.15)$ & $(1.1200,1.6800,0.6000,0.6000)$ \\
\hline
\end{tabular}

The Grey Relational Matrix of coupled tasks is computed through the tearing algorithm: 


$$
\mathbf{R}=\left(r\left(X_{i}, X_{j}\right)\right)_{7 \times 7}=\left[\begin{array}{ccccccc}
1 & 0.5638 & 0.6217 & 0.6048 & 0.5496 & 0.5628 & 0.4588 \\
0.5638 & 1 & 0.8271 & 0.7657 & 0.8686 & 0.7171 & 0.7373 \\
0.6217 & 0.8271 & 1 & 0.8659 & 0.7403 & 0.6286 & 0.6358 \\
0.6048 & 0.7657 & 0.8659 & 1 & 0.7301 & 0.6268 & 0.6673 \\
0.5496 & 0.8686 & 0.7403 & 0.7301 & 1 & 0.7761 & 0.7353 \\
0.5628 & 0.7171 & 0.6286 & 0.6268 & 0.7761 & 1 & 0.6572 \\
0.4588 & 0.7373 & 0.6358 & 0.6673 & 0.7353 & 0.6572 & 1
\end{array}\right] .
$$

Since $\left.r\left(X_{1}, X_{7}\right)\right)$ is minimum, we can firstly cut off the information connection between $T_{1}$ and $T_{7}$. After cutting off a connection every time, reprogram the coupled tasks according to Theorem 1 and 2 . At last, we can obtain the execution order of the 7 coupled tasks: $T_{1} \rightarrow T_{4} \rightarrow T_{2} \rightarrow T_{3} \rightarrow T_{5} \rightarrow T_{6} \rightarrow T_{7}$. Fig. 8 shows the resequenced coupled block by tearing, and the area surrounding by the dashed frame is the sub-block of coupled block, which indicates less loop in it.

\begin{tabular}{|l|l|l|l|l|l|l|l|}
\hline & $T_{1}$ & $T_{4}$ & $T_{2}$ & $T_{3}$ & $T_{5}$ & $T_{6}$ & $T_{7}$ \\
\hline New Product Design & $*$ & & 1 & 1 & & & 1 \\
\hline Drawing of Produce Project & 1 & $*$ & & & & & \\
\hline Technology Analysis & 1 & & $*_{*}$ & 1 & & & \\
\hline Performance Analysis & 1 & & 1 & $*^{\prime}$ & & 1 & \\
\hline New Product Produce & & 1 & 1 & 1 & $*$ & & \\
\hline Performance Test & & & & 1 & 1 & $*$ & \\
\hline New Product Improvement & 1 & & 1 & & & 1 & $*$ \\
\hline
\end{tabular}

Fig. 8. Resequenced coupled block by tearing

In general design, the technology and performance analysis are carried on after the new product design, and then the produce project drawing is made. This design approach is indicated in Fig. 7. However, in Fig. 7, there is a small-scale loop, that is performance analysis-performance test-performance analysis loop shown in the dashed frame, in the large-scale loop new product design-improvement of new product-new product design. Tearing programming can restrict the loop of the dashed frame in a less scope. Fig. 8 shows the resequenced coupled block by tearing. The drawing of produce project is made firstly after the design of new product. Secondly, the technology and performance analysis are carried on. Thus, the loop performance analysis - performance test-performance analysis is restricted in a fewer sub-block shown in dashed frame in Fig. 8. Because of tearing at the weakest point, quality loss will be reduced to the least. At the same time, translate the sub-block into WTM, then use eigenvalue and eigenvector to calculate the total work of it. The formula is as follows:

$$
U=S(I-\Lambda)^{-1} S^{-1} \mu_{0},
$$

where $U$ is the total work of the sub-block, $\Lambda$ is a diagonal matrix of the eigenvalues of the sub-block, $S$ is the corresponding eigenvector matrix, $I$ is the identity matrix, and initial work vector $\mu_{0}$ is a vector of ones which indicates that all of the work remains to be completed on every task at the beginning of the iteration process.

Notice that when translate the sub-block into WTM, off-diagonal entries might be substituted by weak, medium, or strong dependence to describe the estimate of the amount of work, because of the robustness of $\mathrm{WTM}^{[6]}$. Thus, numerical value $0.5,0.25,0.05$ is used to denote strong, medium, and weak dependence, respectively and brings no significant changes to the results. If the period of every activity in WTM is known, the total period of WTM can also be known. Finally, take the sub-block as one element to deal with. After applying these two approaches, the new DSM by reengineering is shown in Fig. 9, where "•" denotes strong dependence, " $\bigcirc$ " denotes medium dependence, " $\triangle$ " denotes weak dependence.

The reengineering of the design process is achieved by substituting a small iteration for a large iteration. Furthermore, comparing with the extended DFD in Fig. 2, the reengineering DSM is the result of refinement 


\begin{tabular}{|l|l|l|l|l|l|l|l|l|l|l|l|l|l|l|l|l|l|l|l|}
\hline & & 1 & 6 & 2 & 3 & 10 & 14 & 15 & 7 & 18 & 16 & 17 & 8 & 9 & 11 & 12 & 13 & 5 \\
\hline Requirement Analysis & 1 & $*$ & & & & & & & & & & & & & & & & \\
\hline Sale Analysis & 6 & & $*$ & & & & & & & & & & & & & & & \\
\hline Sale Project & 2 & 1 & & $*$ & & & & & & & & & & & & & & \\
\hline Design Planning & 3 & 1 & & & $*$ & & & & & & & & & & & & & & \\
\hline Requirement of Performance & 10 & 1 & & & & $*$ & & & & & & & & & & & & \\
\hline Requirement of Time & 14 & & & & 1 & & $*$ & & & & & & & & & & \\
\hline Design and Produce Project & 15 & & & & 1 & & & $*$ & & & & & & & & & & \\
\hline New Product Design & 7 & & & & & 1 & & 1 & $*$ & & & \\
\hline Drawing of Produce Project & 18 & & & & & & & & & & & & \\
\hline
\end{tabular}

Fig. 9. The new DSM by reengineering

of DSM step by step. The design process described by original extended DFD is very abstract, and it can only express the whole design flow. The reengineering DSM is refined from the extended DFD and it can be implemented as a feasible project.

\section{Conclusions}

This paper analyzes DFD and presents extended DFD to describe the complex design flow of a factory. Through transforming the extended DFD graph into information design flow graph, we are able to be convenient to express the product development process on computer. And then we also find out the present optimal design flow by evaluating the possible design information flows and gain top DSM after mapping the present optimal flow to DSM. Finally, the top DSM is refined, and the reengineering of the design flow is achieved through operating and scheduling the refined DSM. In addition, the most difficult step in analyzing and reengineering of the whole product development process is to dispose the coupling of DSM. There is still no excellent strategy. Further studied on the strategies for disposing coupling are needed and necessary.

\section{References}

[1] T. R. Browning. Applying the design structure matrix to system decomposition and integration problem: a review and new direction. IEEE Transactions on Engineering Management, 2001, 48: 292-306.

[2] J. L. Deng. The Foundation of Grey Theory. Huazhong University of Science and Technology Press, Wuhan, 2002.

[3] W. M. Du, J. Davis, M. C. Shan. Flexible specification of workflow compensation scopes. ACM SIGGROUP, Phoenix, 1997, 309-316.

[4] C. A. Ellis, G. J. Nutt. Modeling and enactment of workflow systems. in: Application and Theory of Petri-Nets, Springer-Verlag, Berlin, 1993.

[5] A. Kusiak, T. N. Larson, J. Wang. Reengineer of design and manufacturing processes. Computers and Industrial Engineering, 1994, 26: 521-536. 
[6] R. P. Smith, S. D. Eppinger. Identifying controlling features of engineering design iteration. Management Science, 1997, 43: 276-293.

[7] D. V. Steward. The design structure system: A method for managing the design of complex systems. IEEE Transactions on Engineering Management, 1981, 71-74.

[8] Y. Wang, Y. Xing, X. Y. Ruan. Information modeling and re-engineering for design process. Computer Integrated Manufacturing System, 2002, 8: 111-114.

[9] R. B. Xiao. A new approach to modeling the complex system structure. Process in Natural Science, 2001, 11: 25-32.

[10] R. B. Xiao, S. W. Si. Research on the process model of product development with uncertainty based on activity overlapping. Integrated Manufacturing Systems, 2003, 14: 567-574.

[11] R. B. Xiao, Z. W. Tao, Y. Liu. Principle and Technology of Intelligent Design. Science Press, Beijing, 2006.

[12] Y. F. Xie, G. X. Yang, M. L. Shi. Conditional directed graph-based workflow process re-engineering. Chinese Journal of Computers, 2001, 24: 729-735.

[13] A. Zakarian, A. Kusiak. Process analysis and reengineering. Computers and Industrial Engineering, 2001, 41: 135-150. 